\title{
Heritage marketing e valorizzazione del territorio: il percorso verso l'innovazione sostenibile nel settore tessile e moda
}

\author{
Alberta Bernardi ${ }^{*}$, Chiara Luisa Cantü**, Elena Cedrola ${ }^{* * *}$
}

Ricevuto 20/10/2020 - Accettato 28/01/2021

\section{Sommario}

Le imprese sono chiamate ad innovare i loro processi e prodotti in chiave sostenibile per differenziarsi e raggiungere un vantaggio competitivo. Conciliare gli investimenti in innovazione sostenibile con la redditività aziendale potrebbe rappresentare un problema per molte imprese, ancora incerte su quali ne siano i pro e i contro, ma soprattutto su quali scelte strategiche debbano essere adottate al fine di sviluppare un'innovazione che sia sostenibile, in grado di creare valore per il mercato e per l'impresa. L'obiettivo di questo paper è contribuire al crescente corpus di letteratura che si interroga su cosa realmente sia l'innovazione sostenibile e sui driver che ne consentono l'ideazione e lo sviluppo. Nello specifico, vengono indagate le potenzialità strategiche dell'heritage marketing quale strumento per valorizzare la storia aziendale e i suoi valori, tra cui la sostenibilità e il territorio. Focus della ricerca è il settore tessile e moda a ragione della sua più volte denunciata insostenibilità - a livello ambientale, sociale ed economico - e scarsa apertura verso l'innovazione - nei processi, prodotti e modelli di business. Le evidenze empiriche derivanti dalla presentazione di un caso studio di un'azienda di moda italiana internazionalizzata suggeriscono che la gestione strategica del corporate heritage e del brand heritage (o del patrimonio storico e culturale dell'impresa e dei suoi valori) può consentire alle imprese di promuovere l'innovazione sostenibile. Ciò grazie alla valorizzazione della storia aziendale e ai valori che hanno contraddistinto lo sviluppo

* Ph.D. Candidate in Management e Innovazione. Dipartimento di Scienze dell'economia e della gestione aziendale. Università Cattolica del Sacro Cuore di Milano. alberta.bernardi @, unicatt.it

** Professore Associato. Dipartimento di Scienze dell'economia e della gestione aziendale. Università Cattolica del Sacro Cuore di Milano. chiara.cantu@unicatt.it

*** Professore Ordinario di Economia e Gestione delle Imprese. Dipartimento di Economia e Diritto. Università di Macerata. elena.cedrola@unimc.it

Corporate Governance and Research \& Development Studies, n. 1-2021

(ISSN 2704-8462-ISSNe 2723-9098, DOI: 10.3280/cgrds1-2021 oa10566) 
dell'impresa: sostenibilità ambientale e sostenibilità sociale. Le due forme di sostenibilità spingono a considerare non solo la riduzione dell'impatto della produzione sull'ambiente, ma anche le azioni propositive che un'impresa può compiere nei confronti del territorio e della comunità locale. Emerge anche con forza la necessità per la filiera tessile di orientarsi ad una maggiore, se non totale, circolarità.

Parole chiave: innovazione sostenibile, patrimonio storico e culturale, territorio, caso studio, settore tessile e moda

\begin{abstract}
Heritage marketing and territorial enhancement: the path towards sustainable innovation in the textile industry

Manufacturing companies have to innovate their processes and products sustainably to differentiate themselves and achieve a competitive advantage. Reconciling investment in sustainable innovation with corporate profitability could be a problem for many companies, still uncertain about the pros, cons, and strategic choices required to develop successful sustainable innovation. This article aims to contribute to the growing body of literature that questions what sustainable innovation is and its implementation drivers. Specifically, heritage marketing's strategic potential is investigated as a tool for enhancing the company's history and local resources. The current research focuses on the textile and fashion industry due to its repeatedly denounced unsustainability - at an environmental, social, and economic level - and a lack of openness to innovation - in processes, products, and business models. The empirical evidence, deriving from the presentation of a case study of an internationalized Italian fashion company, suggests that the corporate and brand heritage's strategic management (or the company's historical and cultural heritage and values) can enable companies to implement sustainable innovation. The latter allows firms in value creation and value exchange with the market. There is also a strong need for the textile supply chain to move towards a greater circularity.
\end{abstract}

Keywords: sustainable innovation, heritage marketing, territory, case study, textile and fashion industry

\title{
1. Introduzione
}

Di fronte ai problemi che affliggono il mondo come povertà e inquinamento, due sembrano essere le soluzioni percorribili: sostenibilità ed innovazione. Tuttavia, se la sostenibilità è solo un principio, il progresso e i cambiamenti da esso generati sono, spesso, la causa dell'insostenibilità in cui versiamo. Sebbene la letteratura sia ancora in evoluzione, sembra convergere sul considerare l'innovazione sostenibile - ovvero un'innovazione che tenga 
conto del progresso tecnologico unitamente al bene dell'ambiente e della società (Barbieri et al., 2010) - come la soluzione ai problemi accennati. Non è però così semplice ed immediato svilupparla e implementarla poiché ad essa sono legati ingenti costi ed elevate incertezze. La coesistenza della sostenibilità ambientale e sociale può essere ravvisata nella prospettiva territoriale. Un'impresa che valorizza le risorse tangibili e intangibili del territorio tenderà a ridurre l'impatto della produzione sull'ambiente circostante, a promuovere il know-how della società locale e i valori insiti nell'area geografica.

L'heritage marketing, se considerato in una prospettiva strategica, potrebbe aiutare a conseguire anche una sostenibilità economica. Infatti, l'heritage marketing, generalmente associato alle attività strategiche che valorizzano il patrimonio storico dell'impresa tramite la promozione dell'immagine e della reputazione della stessa, può aiutare un'impresa a mostrare il proprio passato d'eccellenza e a distinguersi sul mercato (Montemaggi e Severino, 2007). Le iniziative di heritage marketing trasmettono l'identità e la personalità dell'impresa e del brand e si rivolgono sia agli stakeholder interni che a quelli esterni (Misiura, 2006; Balmer, 2013).

L'obiettivo di questo paper è indagare le potenzialità strategiche dell'heritage marketing quale strumento che permette di valorizzare la storia aziendale e il territorio, garantendo la possibilità di implementare un'innovazione sostenibile di successo. Focus della ricerca è il settore tessile e moda, tradizionalmente caratterizzato da una limitata capacità di conseguire obiettivi di sostenibilità ambientale, sociale ed economica, come pure da una innovazione nei processi, prodotti e modelli di business. Questi temi hanno riscontrato ancora scarsa attenzione nelle attività di ricerca. In questo contributo viene presentato un caso studio in profondità di un'azienda di moda italiana internazionalizzata, Successori Reda S.p.a., un'eccellenza del tessile Made in Italy, che da sempre ha identificato tra i suoi principi ispiratori la comunità, l'ambiente e il lavoro. Questi sono espressione di valori forti che caratterizzano la definizione della vision, mission e strategia aziendale. L'impresa, ancora oggi, si contraddistingue per prodotti di eccellenza che promuovono i valori aziendali, tra cui il suo territorio di origine.

L'intento che questo paper ha di valorizzare l'importanza e la forza della storia aziendale è ancor più apprezzabile in uno scenario di crisi come quello che ha fatto seguito alla pandemia di Covid-19. Infatti, così come il caso aziendale analizzato insegna, una forte dimensione di corporate e brand heritage non solo è indispensabile per il successo di un brand (Cedrola e Trabaldo Togna, 2020), ma può riuscire anche a garantire la sopravvivenza alle crisi di mercato (Cedrola e Battaglia, 2013). L'articolo presenta nella sezione 2 l'analisi della letteratura; nella sezione 3 la metodologia; nella sezione 4 le evidenze empiriche; le conclusioni e gli sviluppi futuri nella sezione 5 . 


\section{Analisi della letteratura}

\subsection{L'innovazione sostenibile nel settore tessile e moda}

La sostenibilità, così come sottolineato nell'Agenda 2030 dalle Nazioni Unite, appare come una call to action e la soluzione a gravi problemi quali: inquinamento, scarsità di risorse, povertà. Tuttavia, per raggiungere tali risultati è necessario innovare e modificare il modus operandi delle imprese manifatturiere, considerate tra le più inquinanti e insostenibili al mondo, spostando l'attenzione dai soli obiettivi economici a quelli ambientali e sociali (Melander, 2018).

Innovare i processi d'impresa e i prodotti è fondamentale per risolvere $\mathrm{i}$ problemi elencati, sebbene dalla Prima Rivoluzione Industriale ad oggi la ricerca spasmodica del mero progresso economico abbia contribuito a causare la situazione insostenibile in cui versiamo. La letteratura da tempo si interroga su come rendere meno nocivo l'impatto del progresso e, sebbene una soluzione univoca non sia ancora stata trovata, si tende a convergere verso l'idea che un'innovazione sostenibile possa realmente contribuire ad uno sviluppo orientato ad una maggiore sostenibilità senza rinunciare al progresso (e.g. Schaltegger et al., 2017).

Se la sostenibilità è volta a garantire che «le nostre azioni attuali non limitino la gamma di opzioni economiche, sociali e ambientali che avranno a disposizione le generazioni future» (Elkington 1999, p. 21), l'innovazione fa sì che vengano creati nuovi prodotti, processi e modelli di business (Schumpeter, 1934). Ne consegue che l'innovazione sostenibile è l'introduzione di prodotti, processi, metodi di gestione che apportano benefici economici, sociali e ambientali (Barbieri et al., 2010). La letteratura tende, spesso, a focalizzarsi solo sull'aspetto ambientale e a trascurare gli aspetti sociali ed etici dell'attività d'impresa (Moro et al., 2018). A tal riguardo, è interessante analizzare il settore tessile e moda poiché caratterizzato da una supply chain frammentata, internazionalizzata, inquinante e basata, troppo spesso, sullo sfruttamento della forza lavoro in Paesi in via di sviluppo (Battaglia et al., 2014). Inoltre, sebbene l'innovazione di prodotto sembri suscitare un discreto interesse a discapito di quella di processo, ancora pochi studi analizzano le modalità seguite dai fashion brand per sviluppare innovazione (Jin et al., 2019).

Inoltre, poiché grazie alla sostenibilità si ottengono vantaggi economici in termini di risparmi e di maggiore attrattività dei consumatori finali, molte aziende tessili e della moda stanno investendo in tecnologie e innovazioni "green" al fine di ottenere solamente vantaggi economici, e non perché la sostenibilità sia un loro valore fondamentale (Cedrola e Trabaldo Togna, 
2020). Infatti, lo studio citato sottolinea quanto sia importante che la sostenibilità e l'innovazione siano parte integrante del patrimonio storico e culturale d'impresa, veri e proprio valori core, e non strumenti di greenwashing finalizzati a richiamare l'attenzione del cliente senza realizzare un investimento significativo in sostenibilità.

\subsection{La rilevanza del corporate heritage e del brand heritage}

Il corporate heritage, ovvero il patrimonio storico e culturale dell'impresa, si compone di densi e significativi collegamenti tra passato, presente, futuro e, se valorizzato in modo consapevole, è in grado di conferire autenticità all'azienda e di trasmettere fiducia e affinità con i diversi stakeholder (e.g. Napolitano et al., 2018; Garofano et al., 2020). La dimensione di corporate heritage, riferendosi ai valori del passato e al loro contributo per il presente e il futuro (Balmer, 2013), si distingue dalla corporate history che si riferisce solo a descrizioni, celebrazioni e simboli del passato (Burghausen e Balmer, 2014). La letteratura ha identificato nella longevità, nei core values, nei simboli e nella valorizzazione del passato i principali fattori che determinano la rilevanza del corporate heritage e sui quali si fonda l'identità d'impresa (Urde et al., 2007). Come evidenziato da Marino e Montera (2015), la corporate heritage identity si concretizza nei tratti dell'identità organizzativa rimasti sì costanti nel tempo, ma che hanno anche assorbito elementi provenienti da altre identità, come quelle di altri luoghi, istituzioni e culture.

Se da un lato si ha il patrimonio storico dell'impresa, dall'altro c'è il patrimonio storico della marca o brand heritage che, fondandosi sulla storia dell'impresa, rappresenta una sintesi fra gli attributi e i valori della marca, la sua longevità e reputazione. I temi di corporate e brand heritage sono importanti nel settore tessile e moda, e ancor più nel segmento lusso, poiché il patrimonio storico è il miglior mezzo per reinterpretare la tradizione, creando un collegamento significativo tra passato e presente con una forte influenza emozionale (Balmer, 2013). Data la loro rilevanza, sia il corporate che il brand heritage necessitano di essere supportati da attività strategiche che li valorizzino con l'obiettivo di promuovere i valori aziendali che contraddistinguono l'identità dell'impresa. L'insieme di queste attività è definito heritage marketing, i cui principali strumenti sono gli archivi aziendali, i musei e le mostre, le public relations e lo storytelling (Lucci e Sacchi, 2014).

Tuttavia, affinché l'impresa riesca a rafforzare la propria strategia di differenziazione e il proprio posizionamento, l'heritage marketing deve utilizzare questi strumenti e tecniche in modo strategico, per trasmettere l'identità 
e la personalità dell'impresa e del brand alle diverse categorie di stakeholder rilevanti (Balmer, 2013; Napolitano et al., 2018). La valorizzazione del patrimonio storico e culturale permette di presentare e trasmettere l'evoluzione dei valori e dell'identità d'impresa, generando un forte senso di appartenenza tra i clienti interni (Misiura, 2006), e di potenziare il rapporto di fiducia con clienti e stakeholder esterni. L'heritage marketing ha l'obiettivo di presentare il passato d'eccellenza dell'impresa per permetterle di distinguersi sul mercato, di acquisire maggiore forza contrattuale, di mostrare un valore d'appartenenza per i propri dipendenti e stakeholder locali, di valorizzare le tradizioni e le specializzazioni di un territorio. Le opportunità che ne derivano sono maggiormente evidenti sui nuovi mercati internazionali dove una storia prestigiosa ha un forte fascino e un valore importante e distintivo rispetto alla concorrenza (Montemaggi e Severino, 2007).

\subsection{Marketing territoriale e valorizzazione dei "significati" del territorio}

Per valorizzare l'importanza della storia aziendale è fondamentale fare un esplicito riferimento al territorio in cui l'impresa è insediata, poiché sono proprio i valori, le vocazioni e le risorse dello stesso ad influenzare in modo preponderante la storia e l'orientamento d'azienda (Cantù e Bernardi, 2019). La valorizzazione un territorio non è un'operazione semplice. È necessario che le imprese definiscano e perseguano diverse strategie che, ricadendo sotto la definizione di "marketing territoriale", consentono loro di identificare e promuovere i valori e le vocazioni di un territorio e di trarne benefici per il loro brand (Platania, 2014).

L'analisi della relazione tra un'impresa tessile e il territorio di riferimento è importante perché un forte rapporto con il territorio è il primo passo per essere percepiti come sostenibili e responsabili grazie alla creazione di posti di lavoro e all'uso di risorse locali (Fletcher, 2008). Sebbene diverse ricerche abbiano evidenziato come lo sviluppo delle potenzialità del territorio possa essere esaminato secondo un modello statico, cioè indagando le risorse presenti in uno spazio caratterizzato da prossimità geografica (e.g. Malmberg $\mathrm{e}$ Maskell, 2002), altre allargano la prospettiva e sostengono che il territorio possa essere identificato come un sistema dinamico in cui il valore si genera attraverso le relazioni interconnesse tra nodi dello spazio relazionale, che non necessariamente deve coincidere con un specifica area geografica (e.g. Cantù e Bernardi, 2019). Infatti, in un mondo sempre più globalizzato, si affermano relazioni tra stakeholders che travalicano la logica della prossimità geografica statica in favore di una dinamica. A tal proposito, il settore tessile e moda si sviluppa su scala globale e, spesso, si basa sulla produzione 
in Paesi a basso costo del lavoro, sull'uso di materie prime di dubbia provenienza e qualità, sul subappalto delle attività produttive a svariate aziende sparse in tutto il mondo (Aakko e Koskennurmi-Sivonen, 2013), tutte caratteristiche potenzialmente problematiche da un punto di vista d'innovazione sostenibile.

Le aziende tessili e della moda hanno, inoltre, la necessità di attrarre consumatori che sono sempre più informati e che vogliono indossare consapevolmente capi differenziati; l'artigianato può essere un buon modo sia per aggiungere dettagli unici, sia per garantire una migliore qualità in alcune fasi della produzione, anche nelle supply chain globalizzate (Aakko e Koskennurmi-Sivonen, 2013). L'artigianato è una parte importante del know-how dei distretti industriali e consiste in combinazioni di tecniche tradizionali e creatività che riescono a permeare l'intera filiera anche se non si sviluppa interamente in un'unica area geografica (Jin e Cedrola, 2018).

Sulla base delle precedenti considerazioni, le aziende tessili e della moda possono sviluppare innovazione sostenibile di successo, ovvero un'innovazione che apporta benefici ambientali, sociali ed economici, quando hanno un patrimonio culturale forte e radicato. Inoltre, la dimensione di heritage ed una corretta strategia ad essa associata, soprattutto in uno scenario internazionale, possono aiutare l'impresa ed il suo brand ad essere forti e riconoscibili.

\section{Metodologia}

Questo paper si basa su un approccio qualitativo e sull'elaborazione di un caso studio, data la necessità di indagare in modo approfondito $i$ temi esposti (Yin, 2003). L'obiettivo della ricerca è indagare le potenzialità strategiche dell'heritage marketing quale strumento per valorizzare la storia aziendale e i valori del territorio (Napolitano et al., 2018). Per fare ciò, viene analizzato Successori Reda S.p.a. (di seguito Reda), eccellenza italiana e mondiale nella produzione di tessuti e abbigliamento in lana. In particolare, durante le varie crisi economiche che il settore tessile ha attraversato, l'azienda è stata in grado di sopravvivere anche e soprattutto grazie ai forti valori tramandati dalla sua fondazione ad oggi (Cedrola e Trabaldo Togna, 2020). La dimensione di heritage permette all'impresa di essere saldamente ancorata al territorio d'origine - il distretto tessile Biellese - e di espandersi a livello internazionale valorizzando la sua identità. 


\subsection{Selezione del caso e raccolta dati}

L'industria tessile e moda italiana è notoriamente composta da piccole e medie imprese (PMI) a conduzione familiare, perlopiù concentrate in distretti industriali. La scelta di analizzare un'azienda appartenente ad un distretto non è casuale: ogni distretto è definito da valori storici, culturali, sociali ed economici che legano l'identità aziendale al territorio di origine, elemento indispensabile per garantire il progresso e il successo del brand $(\mathrm{Ce}-$ drola e Trabaldo Togna, 2020). Questa ricerca si concentra sul distretto tessile Biellese famoso per i suoi tessuti di lana, composto da 1.513 PMI, molto attivo sul fronte dell'innovazione sostenibile' ${ }^{1}$.

Reda, family business fondata da Carlo Reda nel 1865 a Valle Mosso, oggi è un'eccellenza del Made in Italy. Sebbene l'azienda sia presente in tutto il mondo (1' $82 \%$ del fatturato è dovuto alle esportazioni), continua ad essere fortemente legata al territorio d'origine dove effettua la totalità della sua produzione. L'azienda ha da sempre ha cercato di operare in modo sostenibile adottando politiche di rispetto ambientale e di tutela dei lavoratori, divenendo l'unico lanificio al mondo certificato $\operatorname{EMAS}^{2}$ (Eco-Management and Audit Scheme) e la prima azienda tessile italiana certificata B Corp ${ }^{3}$. Reda, di proprietà della famiglia Botto Poala dal 1919, possiede 3 fattorie in Nuova Zelanda in cui pascolano 30.000 pecore su 33.000 ettari di terreno. L'azienda si presta ad essere analizzata perché è in grado di combinare la tradizione artigianale del territorio con una continua innovazione tecnologica, volta sì a mantenere il vantaggio competitivo raggiunto grazie ai prodotti della migliore qualità, ma anche a proteggere e tutelare l'ambiente e la società. Per raccogliere i dati primari, nel 2019, sono state condotte tre interviste semi-strutturate con domande aperte della durata di un'ora circa ciascuna a cui ha risposto Ercole Botto Poala, CEO dell'azienda. Le interviste sono state registrate, trascritte verbatim e analizzate separatamente dagli autori per evidenziare i concetti chiave. In seguito, i concetti simili sono stati uniti e poi combinati con dati secondari raccolti da altre fonti (sito web e report aziendali). Le interviste hanno seguito il protocollo di ricerca definito

\footnotetext{
${ }^{1}$ La ricerca "Ecodistretti 2009" ha analizzato il rapporto tra politiche ambientali innovative e sistemi aziendali dei distretti italiani e, su 54, il distretto tessile biellese si è classificato dodicesimo (Dansero e Caldera 2013).

${ }^{2}$ Fino ad oggi, l'azienda ha investito $20 \mathrm{mln}$ di euro in questa certificazione (dall'intervista a Ercola Botto Poala)

${ }^{3} \mathrm{~B}$ Corporation è una certificazione ottenuta dalle aziende che volontariamente soddisfano i più elevati standard di trasparenza, responsabilità e sostenibilità. È diffusa in oltre 70 Paesi e viene rilasciata da B Lab, un ente non-profit internazionale. https://bcorporation.eu/ about-b-lab Ultimo accesso 26/11/2020
} 
sulla base delle evidenze emerse dalla letteratura tesa ad esplorare i due macro-temi su cui lo studio si basa: la gestione strategica dell'heritage marketing e l'approccio all'innovazione sostenibile. Le interviste sono state suddivise in due momenti:

1. poiché dall'analisi della letteratura e delle informazioni secondarie si evince che l'heritage marketing può essere un valido strumento per valorizzare la storia aziendale e i valori del territorio, si è deciso di discutere della sua rilevanza con l'intervistato in modo da determinarne l'importanza per l'azienda analizzata;

2. in un secondo momento, è stato chiesto all'intervistato di descrivere il proprio approccio all'innovazione sostenibile e di identificare i driver che ne influenzano maggiormente l'attuazione.

\section{Evidenze empiriche}

Le tre interviste semi-strutturate al CEO di Reda sono state codificate in modo indipendente dagli autori con l'obiettivo di identificarne temi e concetti chiave. Successivamente, le differenze di codifica sono state discusse e riviste di conseguenza. Nella Tab. 1 si riportano i codici identificati e gli estratti delle interviste. Nei paragrafi seguenti, i codici vengono spiegati ed analizzati combinandoli con i dati secondari raccolti.

Sulla base dei codici e degli estratti, di seguito presentiamo i risultati principali della ricerca che sono riconducibili allo sviluppo di un'innovazione sostenibile e alla valorizzazione della storia e del territorio. 
Tab. 1 -Codici e estratti delle interviste

\begin{tabular}{|c|c|}
\hline Codici & Estratti \\
\hline \multirow{2}{*}{ Territorio } & $\begin{array}{l}\text { Gli sforzi di Reda sono sempre stati tesi a preservare la cultura della lana e le } \\
\text { sue tecniche di produzione che provengono da un amore per la terra e da un } \\
\text { profondo rispetto per le sue risorse, motivo per cui l'azienda, nonostante sia } \\
\text { presente in molti paesi nel mondo, ha scelto di continuare a produrre i suoi } \\
\text { tessuti esclusivamente nella provincia di Biella. }\end{array}$ \\
\hline & $\begin{array}{l}\text { Da sempre abbiamo un rapporto privilegiato con il territorio: è proprio l'amore } \\
\text { e la riconoscenza che nutriamo nei confronti dell'ambiente e delle sue risorse } \\
\text { ciò che ci spinge a cercare continuamente nuove soluzioni per una produzione } \\
\text { sostenibile, a ridotto impatto ambientale. }\end{array}$ \\
\hline \multirow{4}{*}{$\begin{array}{l}\text { Storia e cultura } \\
\text { aziendale }\end{array}$} & $\begin{array}{l}\text { Di sicuro Reda è stata capace di far tesoro dei fallimenti, ha sempre visto il } \\
\text { fallimento come il momento migliore per imparare. Infatti, la nostra storia rac- } \\
\text { conta un'evoluzione di successo fondata su grandi lezioni di vita. Vorrei rias- } \\
\text { sumere i tratti più salienti della nostra storia (continua nel paragrafo 4.2). }\end{array}$ \\
\hline & $\begin{array}{l}\text { Il made in Italy è un grande, enorme, vantaggio: è come partire } 200 \text { metri } \\
\text { prima in una gara di } 400 \text { metri ma, ovviamente, è un vantaggio che va alimen- } \\
\text { tato grazie a continui aggiornamenti e innovazioni. }\end{array}$ \\
\hline & $\begin{array}{l}\text { Credo che i fattori che ci hanno condotto all'attuale successo siano la continua } \\
\text { innovazione tecnologica, che ci porta ad utilizzare sempre i migliori macchi- } \\
\text { nari disponibili sul mercato per poter garantire un'ottima qualità. }\end{array}$ \\
\hline & $\begin{array}{l}\text { Altro nostro punto di forza da sempre è la verticalizzazione della supply chain } \\
\text { e il contatto costante con il cliente. Noi crediamo di essere unici, nel senso che } \\
\text { conosciamo la lana come pochissimi altri perché la conosciamo da allevatori, } \\
\text { dalla nascita. }\end{array}$ \\
\hline \multirow{4}{*}{ Innovazione } & $\begin{array}{l}\text { Crediamo che il continuo investimento in nuove tecnologie, macchinari e la } \\
\text { costante ricerca sul prodotto sono i fattori di successo che si riflettono in tutti } \\
\text { i capi prodotti da Reda. }\end{array}$ \\
\hline & $\begin{array}{l}\text { Per noi l'innovazione di processo è essenziale e strettamente legata alla soste- } \\
\text { nibilità. Ė necessario che ogni impresa sia consapevole del suo impatto in ogni } \\
\text { fase della supply chain in modo da poter agire e isolare questo impatto. }\end{array}$ \\
\hline & $\begin{array}{l}\text { Per Reda l'innovazione non è mai fine a sé stessa, ma è sempre tesa a ricercare } \\
\text { una maggiore sostenibilità. Il nostro obiettivo è creare un prodotto che abbia } \\
\text { un impatto non nocivo sull'ambiente e che possa dare un ritorno alla società e } \\
\text { ai nostri lavoratori. }\end{array}$ \\
\hline & $\begin{array}{l}\text { Per noi l'innovazione sostenibile è un valore che riassume anche ciò che ci } \\
\text { siamo detti parlando di innovazione di prodotto e processo. In Reda il cambia- } \\
\text { mento e l'innovazione sostenibile sono al centro di ogni processo, siamo pie- } \\
\text { namente consapevoli della nostra responsabilità nei confronti delle genera- } \\
\text { zioni future. }\end{array}$ \\
\hline Sostenibilità & $\begin{array}{l}\text { Di sicuro la sostenibilità è un trend, ma per noi è un valore che ci è stato tra- } \\
\text { mandato dal nostro fondatore e che noi continuiamo ad annoverare tra i valori } \\
\text { fondamentali. L'impegno profuso da Reda nei confronti dell'ambiente e delle } \\
\text { persone le ha permesso, infatti, di ottenere la certificazione B Corp. }\end{array}$ \\
\hline
\end{tabular}




\begin{tabular}{|c|c|}
\hline & $\begin{array}{l}\text { Per noi il benessere delle persone è al centro di ogni processo. I nostri dipen- } \\
\text { denti sono i protagonisti di un futuro che solo grazie alle piccole azioni quoti- } \\
\text { diane e all'impegno di tutti può essere realmente sostenibile. Per questo mo- } \\
\text { tivo, crediamo nelle persone e poniamo la massima attenzione alle esigenze } \\
\text { sociali, promuovendo un miglioramento costante delle condizioni di lavoro } \\
\text { dei nostri collaboratori. }\end{array}$ \\
\hline & $\begin{array}{l}\text { Per noi sostenibilità è, innanzitutto, trasparenza: l'intera filiera costruita in de- } \\
\text { cenni di attività fa sì che l'azienda oggi abbia un vantaggio competitivo non } \\
\text { indifferente (impossibile da ottenere oggi se non a fronte di ingenti investi- } \\
\text { menti). }\end{array}$ \\
\hline & $\begin{array}{l}\text { La trasparenza è un'arma a doppio taglio: dichiarare il proprio impatto può } \\
\text { avere, infatti, sia un risvolto positivo che uno negativo. Come potrebbe essere } \\
\text { letta una dichiarazione di trasparenza dal consumatore? Che reazione potrebbe } \\
\text { avere sapendo che per produrre un metro quadro di stoffa si usano centinaia di } \\
\text { litri d'acqua e si generano decine di kg di } \mathrm{CO}^{2} \text { ? È probabile che, ad oggi, il } \\
\text { consumatore possa avere una reazione negativa davanti ai numeri dell'impatto } \\
\text { ambientale, ma Reda continua ad essere trasparente perché è convinta che, in } \\
\text { futuro, i consumatori dovranno essere sempre più coscienti. }\end{array}$ \\
\hline Consumatore & $\begin{array}{l}\text { Dal } 2013, \text { l'azienda ha deciso di utilizzare come canale distributivo anche l'e- } \\
\text { commerce. Per noi che facciamo semilavorati è un'opportunità incredibile po- } \\
\text { ter parlare direttamente con il consumatore e trasmettergli i nostri valori. }\end{array}$ \\
\hline \multirow[b]{2}{*}{$\begin{array}{l}\text { Economia } \\
\text { Circolare }\end{array}$} & $\begin{array}{l}\text { Il tema della circolarità è trend attuale a cui tendere nel prossimo futuro. Reda } \\
\text { si rende conto dell'importanza di una supply chain orientata alla circolarità } \\
\text { soprattutto perché la lana è una fibra che costa molto, per cui riutilizzare i } \\
\text { sotto-prodotti della lavorazione è una necessità. Ad oggi, noi riutilizziamo e } \\
\text { vendiamo alle aziende cosmetiche tutta la lanolina (il grasso della pecora, pro- } \\
\text { dotto base per le creme di bellezza) che si ottiene al primo lavaggio della lana. } \\
\text { Altri sotto-prodotti, come i fili corti, li rivendiamo ad altri distretti che si ba- } \\
\text { sano quasi interamente sugli scarti del Biellese. }\end{array}$ \\
\hline & $\begin{array}{l}\text { È chiaro che, a livello economico, il problema maggiore è il capo che rimane } \\
\text { invenduto, poiché è quello che ha assorbito più energia di tutti lungo l'intera } \\
\text { filiera. La sfida è cercare di creare qualcosa che sia anche economicamente } \\
\text { sostenibile. Tuttavia, nonostante i grandi sforzi e l'obiettivo di trasformare la } \\
\text { supply chain da lineare a circolare, l'ostacolo più grande che stiamo incon- } \\
\text { trando è proprio ciò che ci rende emblema di sostenibilità e innovazione, ov- } \\
\text { vero la tracciabilità. Infatti, i prodotti sviluppati da materiali riciclati, spesso, } \\
\text { non danno garanzie circa la provenienza delle materie riciclate. Molti brand } \\
\text { non si curano di questo aspetto perché a loro interessa vendere il prodotto, non } \\
\text { certificare il processo, ma noi non intendiamo sottrarci all'impegno assunto } \\
\text { verso l'ambiente e la società. }\end{array}$ \\
\hline
\end{tabular}

\subsection{L'innovazione sostenibile: una dichiarazione di trasparenza}

Reda è una realtà consolidata a livello internazionale nella fascia lusso del sistema tessile e moda italiano. Alta specializzazione nella produzione, continua innovazione dei processi e dei prodotti, costante attenzione alla qualità e alla creatività, tutela dell'artigianato, miglioramento degli standard dell'ambiente di lavoro per i dipendenti e nel servizio clienti, sono i valori che definiscono il DNA dell'impresa dal 1865. 
Secondo Ercole Botto Poala, il continuo investimento in nuove tecnologie, macchinari e la costante ricerca sul prodotto sono i fattori di successo che si riflettono in tutti i capi prodotti da Reda. Esempio eclatante è Rewoolution, il nuovo brand di Reda che offre abbigliamento tecnico innovativo prodotto al $100 \%$ con lana merino biodegradabile e attraverso tecnologie che minimizzano l'impatto ambientale. Negli ultimi anni, Reda ha impiegato circa $6 \mathrm{mln}$ di euro per ridurre il suo impatto ambientale investendo non solo in ricerca e sviluppo di nuovi materiali, ma anche costruendo un impianto di depurazione che elimina il $99,9 \%$ di sostanze nocive e ricicla circa il $30 \%$ dell' acqua utilizzata. Di conseguenza, Reda riesce ad utilizzare solo 120 litri di acqua per chilogrammo di lana, rispetto ai 500 litri utilizzati dai concorrenti (Dansero e Caldera, 2013).

Oltre a ciò, l'eccellenza di Reda non è solo dovuta alla qualità e all'innovazione dei suoi prodotti e processi, ma anche all'importanza attribuita al benessere sociale e ambientale che si riflette nella sua relazione con i dipendenti e con la società. Quanto detto viene confermato nella mission di Reda: «Come uno dei marchi leader nel settore tessile, abbiamo il potere e la responsabilità di promuovere il cambiamento attraverso l'innovazione sostenibile, la consapevolezza ambientale e il progresso sociale al fine di garantire un futuro stabile e sicuro alle prossime generazioni» ${ }^{4}$.

I tre principi cardine che guidano l'attività dell'impresa sono sintetizzati sul sito web dell'azienda nei seguenti tre punti':

- Le persone al centro:

«Reda pone il benessere delle persone al centro di ogni processo. I nostri dipendenti sono i protagonisti di un futuro che solo grazie alle piccole azioni quotidiane e all'impegno di tutti può essere realmente sostenibile. Per questo motivo, crediamo nelle persone e poniamo la massima attenzione alle esigenze sociali, promuovendo un miglioramento costante delle condizioni di lavoro dei nostri collaboratori».

- Il rispetto per l'ambiente:

«Reda opera in modo responsabile, sostenibile e trasparente non solo nei confronti delle persone, ma anche della comunità e dell'ambiente. Da sempre abbiamo un rapporto privilegiato con il territorio: è proprio questo amore e la riconoscenza che nutriamo nei confronti dell'ambiente e delle sue risorse ciò che ci spinge a cercare continuamente nuove soluzioni per una produzione sostenibile, a ridotto impatto ambientale».

\footnotetext{
${ }^{4}$ https://www.reda1865.com/it/reda-mission Ultimo accesso 26/11/2020

5 http://reda1865.it/bcorp.html\#page2 Ultimo accesso 26/11/2020
} 
- Innovazione sostenibile:

«Reda pone il cambiamento e l'innovazione sostenibile al centro di ogni processo, pienamente consapevole della propria responsabilità nei confronti delle generazioni future. Cruciale è la spinta all'innovazione tecnologica volta a trovare soluzioni all'avanguardia che permettano di realizzare tessuti performanti e unici, nel pieno rispetto dell'ambiente».

Un altro valore importante è la trasparenza (Tab. 1). La dichiarazione di trasparenza a cui ha fatto cenno il CEO di Reda è l'emblema dell'interesse che l'azienda ha per la sostenibilità ambientale e sociale, poiché si rivolge direttamente ai suoi consumatori e stakeholder per assicurare e comunicare loro la qualità e il luogo di produzione dei tessuti. In questa dichiarazione volontaria, la società sottolinea che tutti i tessuti da loro commercializzati sono il risultato di un processo produttivo realizzato completamente in Italia.

Reda riesce a rimanere fedele a una tale dichiarazione grazie ad un'altra componente fondamentale del suo DNA: l'integrazione verticale. Ciò consente all'azienda di conoscere e supervisionare la qualità dell'intera catena di approvvigionamento, dalle fattorie in Nuova Zelanda ai punti vendita dove si ha un contatto diretto con il consumatore finale. Anche la customizzazione dell'offerta è importante perché i consumatori di oggi vogliono indossare consapevolmente capi differenziati. Poiché l'artigianato permette sia di aggiungere dettagli unici sia di garantire una migliore qualità in alcune fasi della produzione (Aakko e Koskennurmi-Sivonen, 2013), per Reda è fondamentale valorizzare il knowhow del distretto, la cultura locale e la tradizione artigianale. Infatti, è proprio il territorio a detenere le tradizioni che ispirano l'artigianato che, a sua volta, influenza profondamente l'evoluzione della cultura locale (Jin e Cedrola, 2018).

\subsection{La valorizzazione della storia e del territorio}

La mission di Reda rende evidente quanto l'azienda consideri rilevante il suo ruolo di leader per plasmare e indirizzare un cambiamento positivo nel settore, volto ad innovare in modo sostenibile, dando centralità al benessere ambientale e sociale odierno e futuro, alla qualità, alla dedizione e allo sviluppo culturale della comunità locale. In ogni distretto industriale risiedono profondi valori che legano l'identità di un'azienda al suo territorio di origine e le garantiscono il progresso e il successo (Cedrola e Trabaldo Togna, 2020), ma questo è possibile solo se vengono implementate appropriate strategie di heritage marketing. Le interviste hanno mostrato che la scelta di operare in modo innovativo e sostenibile deriva direttamente dalla cultura dell'azienda e dalla sua identità, e solo in un secondo momento dal fatto che 
ciò possa rappresentare un vantaggio competitivo. Il fondatore di Reda aveva una visione imprenditoriale forte e di lunga durata basata su un solido patrimonio culturale; tale visione è stata rafforzata e preservata fino ad oggi dal nuovo assetto proprietario.

Per un'azienda valorizzare le risorse tangibili (materie prime, prodotti, ecc.) e intangibili (know-how, storia e tradizioni, cultura, ecc.) del territorio diviene una modalità per implementare una strategia orientata alla sostenibilità, considerando quest'ultima non solo con la finalità di ridurre l'impatto della produzione sull'ambiente ma, da un punto di vista sociale, riconoscendo anche un nuovo ruolo dell'impresa nella comunità. Inoltre, la produzione su un territorio crea posti di lavoro e utilizza risorse locali (Fletcher, 2008) consentendo alla stessa comunità di crescere. Tuttavia, essendo il tessile un'industria su scala globale, il suddetto legame con il territorio potrebbe venir meno, così come la qualità e l'eccellenza della tradizione. Per superare questi problemi, le aziende del distretto generalmente preferiscono l'esportazione alla produzione estera, poiché il know-how e l'artigianato sviluppati sul territorio sono fonti di vantaggio competitivo. Reda, infatti, ha scelto di esportare e mantenere l'intera produzione nello stabilimento originario, ritenendo che il know-how e le competenze degli artigiani locali svolgano un ruolo chiave nella creazione di tessuti di qualità (Tartaglione et al., 2012).

Tali intenzioni necessitano di essere valorizzate, condivise e raccontate ai clienti interni ed esterni tramite strategie $a d$ hoc. Per questa finalità Reda ha creato sul suo sito web una sezione denominata "Heritage" 6 dove, grazie ad uno storytelling molto articolato, presenta la sua storia, i suoi valori e il suo legame indissolubile con il passato e con il territorio. A tal proposito, si riportano alcuni estratti tratti dal sito web e rinvenibili al link riportato nella nota a piè di pagina $n .6$ :

"il forte legame con il territorio è uno dei tratti distintivi di Reda (...). Qualità e incessante ricerca stilistica si uniscono in un tessuto che interpreta e rivisita i disegni sartoriali classici per farsi espressione più pura e autentica dell'artigianalità e del gusto italiano".

\section{E ancora,}

"se c'è una cosa che dal 1865 ad oggi non è mai cambiata, è il forte legame con il territorio, una vocazione che nasce dal profondo rispetto per le origini territoriali e da una tradizione artigianale secolare, tramandata di generazione in generazione. Quella della lana è infatti una cultura profondamente radicata nella manualità degli artigiani locali, che da sempre contribuiscono al successo dei tessuti dello storico lanificio".

\footnotetext{
${ }^{6}$ https://www.reda1865.com/it/made-in-italy Ultimo accesso 26/11/2020
} 
La passione di Reda per il suo territorio e il suo lascito culturale è emersa anche durante le interviste. Di seguito si riporta la storia dell'azienda così come ci è stata raccontata dal suo CEO. Da questa sintesi emerge nuovamente l'importanza rivestita dal suo patrimonio storico culturale, ovvero la guida all'innovazione, alla sostenibilità e alla valorizzazione del territorio, la cui forza si è rivelata anche in presenza di situazioni critiche:

"Nel 1968 un'alluvione ha distrutto il nostro stabilimento, ma siamo stati capaci di trasformare una tragedia in un'opportunità. Abbiamo ricomprato tutti i macchinari, questa volta al passo con la tecnologia. Da quel momento, l'azienda ha continuato ad investire costantemente in tecnologia al punto che oggi è considerata una delle aziende italiane più innovative. L'innovazione è non è stata soltanto technology driven ma orientata fin da subito a una gestione attenta delle relazioni con i business partner, considerati quali interlocutori strategici per offrire una proposta di valore. Nel 1993 i nostri grandi competitor intuiscono il potere dei consumatori finali, quindi iniziano a rafforzarsi a valle. Reda, invece, si rafforza a monte acquisendo fattorie di Nuova Zelanda; l'ossessione della qualità e l'amore per una profonda conoscenza delle materie prime spinge i Successori Reda a diventare allevatori. Nel 1998 Reda costruisce da capo uno stabilimento seguendo delle logiche innovative volte a causare il minimo impatto possibile. L'importanza di un investimento del genere è tanto più evidente oggi: nel 1998 nessuno parlava di green e sostenibilità, ma Reda costruisce con queste logiche (spendendo molti più soldi di quelli che avrebbe speso altrimenti) perché l'innovazione, la sostenibilità e il pieno rispetto della comunità circostante sono parte del DNA dell'azienda dal 1865 . Venti anni dopo questa scelta si è rivelata essere un tool di marketing al passo con i tempi (...) Nel 2008 la crisi mondiale causa un calo drastico del fatturato, ma Reda trova la sua forza nel continuare ad investire per il miglioramento"7.

\subsection{Economia circolare}

Negli ultimi anni, l'economia circolare ha acquisito un forte impulso nel settore tessile, dove molte aziende si stanno impegnando in varie iniziative post-vendita volte a generare valore anche dai capi d'abbigliamento dismessi (Pal, 2016), e ad acquisire vantaggio competitivo mostrandosi responsabili e innovativi agli occhi dei consumatori. L'economia circolare è l'evoluzione da un sistema lineare, basato sullo sfruttamento continuo di materie prime, a un modello closed loop, ovvero progettato per rigenerare e riutilizzare le risorse e gli scarti di produzione in modo virtuoso (Strähle e Matthaei, 2017; Cedrola e Trabaldo Togna, 2020).

\footnotetext{
${ }^{7}$ Estratto dell'intervista a Ercole Botto Poala, CEO di Successori Reda S.p.a.
} 
Il tema della circolarità emerge dalle interviste a Reda come un trend attuale a cui tendere nel prossimo futuro. Reda si rende conto dell'importanza di una supply chain orientata alla circolarità soprattutto perché la lana è una fibra che costa molto, per cui riutilizzare i sotto-prodotti della lavorazione è una necessità. Ad oggi, Reda vende alle aziende cosmetiche la lanolina che ottiene al primo lavaggio della lana, mentre rivende altri sotto-prodotti, come i fili corti, ad altri distretti (Tab. 1).

Anche per Reda, a livello economico, il problema maggiore è chiaramente rappresentato dal capo che rimane invenduto, poiché è quello che ha assorbito più energia di tutti lungo l'intera filiera. La sfida è cercare di creare qualcosa che sia anche economicamente sostenibile. Tuttavia, nonostante i grandi sforzi e l'obiettivo di trasformare la propria supply chain da lineare a circolare, l'ostacolo più grande che Reda sta incontrando è proprio ciò che la rende emblema di sostenibilità e innovazione, ovvero la tracciabilità. Infatti, i prodotti sviluppati da materiali riciclati, spesso, non danno garanzie circa la provenienza delle materie riciclate. Molti brand non si curano di questo aspetto perché a loro interessa vendere il prodotto, non certificare il processo, ma Reda non intende sottrarsi all'impegno assunto verso l'ambiente e la società.

\section{Conclusioni e sviluppi futuri}

\subsection{Implicazioni teoriche}

Riviezzo et al. (2016) hanno evidenziato che le imprese longeve, nonostante vengano ritenute in grado di mantenere un vantaggio competitivo grazie alle opportunità che la loro storia gli conferisce, troppo spesso non fruttano le potenzialità del loro patrimonio storico e culturale. Reda - fondata nel tardo ' 800 - si discosta da tale evidenza. L'azienda non ha mai smesso di tendere ad un livello superiore di eccellenza, grazie alla costante combinazione della ricerca di una piena sostenibilità ambientale e sociale con l'innovazione di processo e di prodotto. L'azienda ha da sempre investito nella definizione di nuovi processi produttivi e nel lancio di nuovi prodotti orientati ad una maggiore sostenibilità ambientale. Al tempo stesso, Reda è un caso emblematico di sostenibilità sociale, in quanto è in grado di valorizzare la comunità in cui opera e il territorio di riferimento. In particolare, presta una rilevante attenzione alla gestione della relazione con i propri clienti interni (ovvero il personale) interessando nell'attività lavorativa la comunità locale. Tale comunità è stata coinvolta anche attraverso le relazioni di business con le imprese del territorio, determinando lo sviluppo dell'economia 
locale e delle relative tipicità, facendo leva sul know-how unico che la tradizione e l'artigianalità del distretto possono assicurare.

La valorizzazione del territorio e delle sue tipicità e, al tempo stesso, della storia aziendale che li identifica come valori, suggeriscono le potenzialità strategiche del corporate e del brand heritage per lo sviluppo dell'innovazione sostenibile. Se il patrimonio culturale e storico del brand viene considerato dall'impresa quale driver per il lancio di nuovi prodotti e la definizione di nuovi processi, sarà possibile per la stessa impresa creare una soluzione unica e autentica, di maggior valore per la clientela. Questi risultati sono in linea con la letteratura che afferma che la storia aziendale, se valorizzata in chiave strategica, è un elemento di distintività che permette alle aziende di emergere e distinguersi rispetto ai competitor (Napolitano et al., 2018).

La relazione impresa-territorio riconosce la centralità delle risorse territoriali, tangibili e intangibili, che possono contribuire sia alla definizione della corporate identity che all'ideazione di nuovi prodotti e nuovi processi. Al tempo stesso, tale relazione è sempre più indagata considerando la capacità dell'impresa di promuovere le specificità territoriali. La dimensione di corporate e brand heritage è, pertanto, legata ad una gestione strategica dei valori aziendali, tra cui il territorio e la sua promozione. Attraverso la conoscenza della storia aziendale e dell'evoluzione del sistema produttivo, è possibile comprendere e riscoprire le peculiarità dell'impresa e del territorio che la ospita.

La promozione del territorio e dei valori dell'impresa avviene attraverso lo storytelling, ovvero attraverso la diffusione della conoscenza della storia dell'impresa. Lo storytelling, che in Reda si concretizza nella valorizzazione della cultura aziendale e nella dichiarazione di trasparenza e tracciabilità volontaria, è in grado di trasformare i possibili punti di debolezza (come il consumo di risorse nel processo produttivo) in punti di forza. Lo storytelling, essendo uno degli strumenti principali dell'heritage marketing, diventa un driver strategico per promuovere il brand, l'impresa e i suoi valori. Come evidenziato da alcuni autori (e.g. Wilson, 2018), l'heritage marketing consente di rafforzare l'identità distintiva del brand e di creare fiducia nei suoi confronti. Oltre a ciò, come emerso dal caso studio, la strategia di heritage marketing, sviluppata attraverso strumenti offline e online, permette di rafforzare la relazione con i clienti e con gli altri stakeholder, condividendo con loro i valori aziendali e del territorio. Infine, dall'analisi emerge con forza la necessità per la filiera tessile di orientarsi ad una maggiore, se non totale, circolarità, definendo una chiara strategia per raggiungere tale obiettivo. 


\subsection{Implicazioni manageriali}

Oggi il tema della sostenibilità assume un rilievo crescente per le imprese che appartengono a diversi settori, in particolar modo per gli attori del settore tessile e moda. Lo sviluppo di una strategia sostenibile, che si differenzia dalle azioni di greenwashing, richiede alle imprese investimenti significativi, di lungo termine. In tale prospettiva, lo sviluppo di un'innovazione di prodotto sostenibile richiede l'introduzione di nuovi processi green-oriented. Nel contempo, la sostenibilità non deve limitarsi alla sfera ambientale. Come evidenziato dal caso studio, è possibile combinare sostenibilità ambientale $\mathrm{e}$ sostenibilità sociale con riferimento non solo alle azioni che un'impresa può compiere nei paesi con situazioni critiche, ma anche alla gestione delle relazioni con i clienti interni (ovvero il personale) e con le imprese del territorio per valorizzare le risorse locali, tangibili e intangibili.

In particolar modo, quando la sostenibilità viene considerata quale valore core di un'impresa e della sua storia, la sua valorizzazione e promozione può essere sviluppata attraverso una strategia di heritage marketing. La conoscenza della storia di un'impresa comporta anche la conoscenza dei suoi valori e dei valori del suo territorio. Questa considerazione, in una prospettiva più ampia, ci consente anche di riconsiderare il ruolo dell'impresa nella società. La finalità dell'impresa non è solo la creazione di un valore economico, ma anche di un valore per la società che viene co-creato con la stessa e con il territorio.

\subsection{Limiti e sviluppi futuri}

Limiti di questo studio sono riscontrabili nella considerazione di un unico caso studio, nonostante la sua rilevanza nel panorama tessile italiano e internazionale. Futuri studi potrebbero utilizzare le considerazioni qui presentate comparando, con un multiple case study, più aziende appartenenti anche a distretti e settori differenti (Riviezzo et al., 2016; Garofano et al., 2020). L'integrazione con un approccio quantitativo, inoltre, potrebbe consentire di misurare l'efficacia della strategia di heritage marketing.

\section{Riferimenti bibliografici}

Aakko M., Koskennurmi-Sivonen R. (2013). Designing sustainable fashion: Possibilities and challenges. Research Journal of Textile and Apparel, 17(1): 13-22. DOI: 10.1108/RJTA-17-01-2013-B002 
Balmer G.M.T. (2013). Corporate heritage, corporate heritage marketing, and total corporate heritage communications. Corporate Communications: An International Journal, 18(3): 290-326. DOI: 10.1108/CCIJ-05-2013-0031

Barbieri J.C., Vasconcelos I.F.G.D., Andreassi T., Vasconcelos F.C.D. (2010). Innovation and sustainability: new models and propositions. Rae-Revista de Administração de Empresas, 50(2): 146-154. DOI: 10.1590/s0034-75902010000200002

Battaglia M., Testa F., Bianchi L., Iraldo F., Frey M. (2014). Corporate social responsibility and competitiveness within SMEs of the fashion industry: Evidence from Italy and France. Sustainability, 6(2): 872-893. DOI: 10.3390/su6020872

Burghausen M., Balmer J. M. (2014). Corporate heritage identity management and the multi-modal implementation of a corporate heritage identity. Journal of Business Research, 67(11): 2311-2323. DOI: 10.1016/j.jbusres.2014.06.019

Cantù C., Bernardi A. (2019). Marketing territoriale: lo sviluppo di potenzialità innovative latenti attraverso il networking. Micro \& Macro Marketing, 28(1): 159184. DOI: $10.1431 / 93021$

Cedrola E., Battaglia L. (2013). Country-of-origin effect and firm reputation influence in business-to-business markets with high cultural distance. Journal of Global Scholars of Marketing Science,23(4): 394-408. DOI: $10.1080 / 21639159.2013 .818280$

Cedrola E., Trabaldo Togna L. (2020). Eccellenze italiane. Internazionalizzazione ed ecosostenibilità del distretto tessile Biellese. Milano: Pearson.

Dansero E., Caldera G. (2013). Green Economy e tessile: chi passa per la cruna dell'ago? In: Ferlaino F., Bagliani M., Crescimanno A., Nepote D., a cura di, $L a$ green economy in Piemonte. Rapporto Ires 2013. Torino: Ires Piemonte.

Elkington J. (1999). Cannibals with Forks: The Triple Bottom Line of 21st Century Business. Oxford: Capstone.

Fletcher K. (2008). Sustainable fashion \& textiles: design journeys, Earthscan. New York: Sterling.

Garofano A., Riviezzo A., Napolitano M.R. (2020). Una storia, tanti modi di raccontarla. Una nuova proposta di definizione dell'heritage marketing mix. Il Capitale Culturale. Studies on the Value of Cultural Heritage, 10: 125-146. DOI: $10.13138 / 2039-2362 / 2460$

Jin B., Cedrola E., a cura di (2018). Product Innovation in the Global Fashion Industry. New York: Palgrave Macmillan.

Jin B.E., Cedrola E., Kim N.L. (2019). Process Innovation: Hidden Secret to Success and Efficiency. In: Jin B., Cedrola E., a cura di, Process Innovation in the Global Fashion Industry. New York: Palgrave Macmillan.

Lucci P., Sacchi S. (2014). Brand Jamming. Heritage marketing, co-branding, brand extension: l'evoluzione del branding. Milano: Franco Angeli.

Malmberg A., Maskell P. (2002). The elusive concept of localization economies: Towards a knowledge-based theory of spatial clustering. Environment and Planning, 34: 429-449. DOI: 10.1068/a3457

Marino V., Montera R. (2015). Corporate Heritage e Web Communication per le imprese del Made in Italy. 14th International Congress Marketing Trends Paris, January 23-24. 
Melander L. (2018). Customer and supplier collaboration in green product innovation: External and internal capabilities. Business Strategy and the Environment, 27(6): 677-693. DOI: $10.1002 /$ bse.2024

Misiura S. (2006). Heritage Marketing. Oxford: Elsevier.

Montemaggi M., Severino F. (2007). Heritage Marketing. La storia dell'impresa italiana come vantaggio competitivo. Milano: Franco Angeli.

Moro R.D.C.L., Mendes F.D., Neto J.A. (2018). Sustainable Development in the Supplier Chain: Analysis of a Brazilian Fashion Retailer's Social Responsibility Program. In: Chow P.S., Chiu C.H., Yip A.C., Tang A.K., a cura di, Contemporary Case Studies on Fashion Production, Marketing and Operations. New York: Springer.

Napolitano M.R., Riviezzo A., Garofano A. (2018). Heritage Marketing. Come aprire lo scrigno e trovare un tesoro. Napoli: Editoriale Scientifica.

Pal R. (2016). Sustainable value generation through post-retail initiatives: an exploratory study of slow and fast fashion businesses. Green fashion: 127-158. Singapore: Springer.

Platania S. (2014). Marketing territoriale e valorizzazione del prodotto tipico locale: una ricerca nel contesto siciliano. Annali della Facoltà di Scienze della Formazione, Università degli Studi di Catania, 12: 133-145. DOI: 10.4420/unictasdf.12.2013.8

Riviezzo A., Garofano A., Napolitano M.R. (2016). "Il tempo è lo specchio dell'eternità". Strategie e strumenti di heritage marketing nelle imprese longeve italiane. Il Capitale Culturale. Studies on the Value of Cultural Heritage, 13: 497523. DOI: 10.13138/2039-2362/1360

Schaltegger S., Etxeberria I.Á., Ortas E. (2017). Innovating corporate accounting and reporting for sustainability-attributes and challenges. Sustainable Development, 25(2): 113-122. DOI: 10.1002/sd.1666

Schumpeter J. (1934). Capitalism, Socialism, and Democracy. New York: Harper \& Row.

Strähle J., Matthaei F.S. (2017). The value chain of a branded second hand storepossible activities to be integrated by a conventional fashion brand. In: Strähle J. (eds) Green Fashion Retail. Springer Series in Fashion Business. Springer, Singapore. 175-198. DOI: 10.1007/978-981-10-2440-5_10

Tartaglione C., Gallante F., Guazzo G., a cura di (2012). Sostenibilità: Moda. Cosa significa, Come si applica, Dove sta andando l'idea di sostenibilità nel sistema moda. Roma: Ares 2.0.

Urde M., Greyser S.A., Balmer J.M. (2007). Corporate brands with a heritage. Journal of Brand Management, 15(1): 4-19. DOI: 10.1057/palgrave.bm.2550106

Wilson R. T. (2018). Transforming history into heritage: applying corporate heritage to the marketing of places. Journal of Brand Management, 25(4): 351-369. DOI: 10.1057/s41262-017-0087-8

Yin R.K. (2003). Case study research: design and methods. Thousand Oaks: Sage. 